\title{
LncRNA CRNDE Promoted Colorectal Cancer Cells Reisitance To Paclitaxel Through Wnt/ $\beta$-Catenin Signaling Pathway
}

\section{Rui Ma}

Ningxia Medical University

Chuan-yang Yu

Ningxia Medical University

\section{Xiang Tao}

Ningxia Medical University

\section{Zhi Yang}

Guangzhou First people's Hospital

Qi Huang

The Biochip Research Center of General Hospital of Ningxia Medical University

\section{Pei-dong You}

Ningxia Medical University

Jin-hai Tian

The Biochip Research Center of General Hospital of Ningxia Medical University

Jia Cao

The Biochip Research Center of General Hospital of Ningxia Medical University

Jie Cao

Guangzhou First people's Hospital

Li-bin Wang ( $\triangle$ wanglibin007@126.com )

The Biochip Research Center of General Hospital of Ningxia Medical University

\section{Research Article}

Keywords: Long non-coding RNA CRNDE, Colorectal cancer cells, Wnt/beta-catenin signaling pathway, Paclitaxel

Posted Date: October 5th, 2021

DOl: https://doi.org/10.21203/rs.3.rs-858244/v1

License: (c) (1) This work is licensed under a Creative Commons Attribution 4.0 International License. Read Full License 


\section{Abstract \\ Background}

The IncRNA colorectal neoplasia differentially expressed (IncRNA CRNDE) is commonly over-expressed in different human cancers and involved in different biological functions. Paclitaxel(PTX) is a tricyclic diterpenoid compound which often used as a natural anticancer drugs in cancer treatments. Although there have many research reports about the mechanisms of LncRNA involved in PTX treatment, there are no any research about IncRNA CRNDE and PTX resistance in colorectal cancer. The purpose of this study is to investigate the mechanisims of LncRNA CRNDE involving PTX resistance in colorectal cancer.

\section{Results}

We constructed IncRNA CRNDE over-expression vector and transfected it into SW620 cell. CCK8, Transwell experiments proved that over-expression of Inc CRNDE increased SW620 cells proliferation and invasion, while the si-CRNDE group was significantly decreased. over-expression CRNDE can significantly up-regulate $\beta$-catenin, c-myc, APC and Axin2 expression and affect the expression of cyclinD1 and CDK4 after treated with PTX.

\section{Conclusion}

IncRNA CRNDE promotes CRCs proliferation, invasion and migration. Over-expression of LncRNA CRNDE enhanced the reisitance of CRC to PTX through inhibition of Wnt/ $\beta$-catenin signaling pathway.

\section{Background}

Colorectal cancer(CRC) is the most commonly occurring cancer worldwide. The occurrence of CRC is affected by age, living habits and regional factors. The prevalence of CRC in developed countries is significantly higher than that in developing countries[1]. According to relevant statistics, there were more than 1.8 million new patients with CRC worldwide and about 880,000 patients died of colorectal cancer[2]. Despite the continuous improvement of medical treatments like surgery, chemotherapy and irradiation combined therapy, the recurrence as well as mortality rate of CRC still at a high level[3]. Therefor, to understanding the molecular mechanisms underlying CRC tumorigenesis and drug resistance and to formulating new therapeutic strategies for CRC become important issue for current research.

Long non-coding RNAs (IncRNAs) is a class of non-coding RNAs which composed of more than 200 nucleotides and not code proteins[4]. IncRNAs was found in various organisms and involved in different biological functions like embryo development, lipid metabolism, and bone anabolism[5]. Several studies have showed that the abnormal expression of IncRNAs in different tumors is tightly related to the tumor occurrence and development[6]. LncRNA colorectal neoplasia differentially expressed (IncRNA CRNDE) is 
located at human chromosome 16 (16q12.2). It initially discovered by colorectal cancer sequencing, the full length of its cDNA was $10327 \mathrm{bp}$, containing 6 exons and 5 introns[7]. Tang et al reported that IncRNA CRNDE plays an oncogenic role in hepatocellular carcinoma (HCC) and promoting cell proliferation and migration through IncRNA CRNDE/miR-337-3p/SIX1 axis[8]. Bai et al found that IncRNA CRNDE could influence cervical tumor growth by sponging miR-183 and regulated CCNB1 expression[9]. Cui et al proved that knockdown of LncCRNDE inhibited wnt/ $\beta$-catenin signaling pathway and increased expression of miR-181a-5p, thus induced CRC cells chemoresistance to 5-Fu or Oxa[10].

Wnt/ $\beta$-catenin signaling pathway is a conserved signaling pathway composed of 19 glycoproteins and plays an important regulatory roles in human cancers. Activated the pathway leads to $\beta$-catenin transfer into nucleus and changed of multiple cellular processes that are associated with cancer cells EMT transition, metastasis and thereby influence cancer progression[11]. Several studies have shown that a variety of cancer are associated with changes in the wnt/ $\beta$-catenin signaling pathway. Huang et al reported that SOX9 promoted non-small-cell lung carcinoma migration, invasion and the EMT process through the Wnt/ $\beta$-catenin pathway[12]. Chen et al found that SALL4 activited wnt/ $\beta$-catenin signaling pathway through regulating the CTNNB1 expression and promoted squamous cervical carcinoma cells proliferation and tumor formation[13].

Paclitaxel(PTX) is often used as the first-line anticancer drug for treatment of various malignant diseases[14]. Although there have many research reports about the mechanisms of PTX in treatment of cancer, the cancer cells resistance to PTX still is a great obstacle in clinical applications[15]. The purpose of this study was to investigate the potential mechanisims of LncRNA CRNDE in colorectal cancer, and to identify the mechanisims of LnCRNA CRNDE involved in PTX resistance in CRC.

\section{Materials And Methods}

\section{Sample collection}

A total of 30 paired colorectal cancer tissues and paracancerous tissues were collected from the Colorectal Surgery Department of the General Hospital of Ningxia Medical University.

The tissue samples were stored in a cryopreservation tube and placed in a liquid nitrogen tank immediately after surgery, and then transferred to a $-80^{\circ} \mathrm{C}$ refrigerator for cryopreservation. The patient did not receive radiation or chemotherapy prior to surgery. All tissue samples were histologically certified by two pathologists with at least five years of experience.

\section{Cells and Cell Culture}

Normal human colon cell line NCM460 and human colon cancer cell lines HT116,SW620,SW480 and LOVO were purchased from the American Typical Cultures Preservation Center (ATCC). The cells were cultured at $37^{\circ} \mathrm{C}$ with $5 \% \mathrm{CO}_{2}$ with Leibovitz's- 15 medium (Gibco,USA) including $10 \%$ fetal bovine serum(FBS, Gibco) and $1 \%$ antibiotics $(100 \mathrm{U} / \mathrm{mL})$. 


\section{RNA extraction and quantitative real time-PCR}

Total RNA was isolated from tissues and cells using TRIzol reagent (Ambion,USA). cDNA was synthesized from mRNA by Applied Biosystems according to the instructions of TAKARA reverse transcription kit. $\beta$-actin was selected as internal standards, and the primer sequences are shown in Table 1. The sequences of all primers were searched in Primerbank database and synthesized by Shanghai Sangon Bioengineering Co., Ltd. RT-qPCR amplification procedure was: $95^{\circ} \mathrm{C}$ for 5 s, annealing at $60^{\circ} \mathrm{C}$ for 30 s, extension at $72^{\circ} \mathrm{C}$ for 30 s, a total of 40 cycles. The relative gene expression of LncRNA CRNDE was calculated using the $2^{-\Delta \Delta \mathrm{Ct}}$ method.

Table 1

Sequences of primers.

\begin{tabular}{|c|c|c|}
\hline Name & & Sequence(5'-3') \\
\hline \multirow[t]{2}{*}{ CRNDE } & Forward & 5'-TGGCTATTGGAAGGAGGAGA-3' \\
\hline & reverse & 5'-CCAGTGGCATCCTACAAGAGA-3' \\
\hline \multirow[t]{2}{*}{$\beta$-actin } & Forward & 5'-CCACGGCTGCTTCCAGCTCC-3' \\
\hline & reverse & 5'-GGACTCCATGCCCAGGAAGGAA-3' \\
\hline
\end{tabular}

\section{Cell transfection}

The LncRNA CRNDE overexpression and inhibition lentiviral vector was synthesized by Jimon Biotechnology Co., Ltd. SW620 cells were cultured in Leibovitz's-15 Medium complete Medium. When the confluence of SW620 cells reached $50 \% \varangle 60 \%$, the culture medium was replaced with basal medium without FBS and antibiotics. Added appropriate amount of transfection agent hitransg A and lentivirus liquid and continue cultured at $37^{\circ} \mathrm{C}$ and $5 \% \mathrm{CO}_{2} .24 \mathrm{~h}$ later, the transfection status was observed under fluorescence microscope, and the basic medium was changed to complete medium for further culture. When the cell density reached more than $80 \%$, the culture was transferred to 6-well plate and finally transferred to T25 culture flask for culture.

\section{Cell counting kit-8(CCK-8) assay}

$100 \mu$ l of lentivirus transfected SW620 cells $\left(1 \times 10^{5} / \mathrm{mL}\right)$ were seeded into 96-well microplates. Each group consisted of 5 parallel wells. When the confluence rate reached about $50 \%-60 \%$, the medium was abandoned, Then, added $10 \mu \mathrm{l}$ of CCK-8 reagen(Glomax, Promega) and continue culture $2 \mathrm{~h} \otimes 4 \mathrm{~h}$. The OD value of each well was detected at $450 \mathrm{~nm}$ wavelength light-emitting detector.

\section{Wound-healing assay}

Different groups of SW620 cells were cultured in six-well plate, when the cell density reached over 70\%, the adherent cell layer was cut with $200 \mu \mathrm{L}$ pipetting tip along the middle of each well. The cells was washed with PBS and the floating cells being removed. After being incubated at $37^{\circ} \mathrm{C}$ in $5 \% \mathrm{CO}_{2}$ 
humidified incubator for $0 \mathrm{~h}, 24 \mathrm{~h}, 48 \mathrm{~h}$ and $72 \mathrm{~h}$, a photo of the wound was taken with a digital camera system Agilent Cary 610. Cell growth images were recorded respectively, and the migration area of the cells in each group was calculated.

\section{Cell cloning experiment}

$3 \times 10^{3}$ per well transfected SW620 cells were cultured at $37^{\circ} \mathrm{C}, 5 \% \mathrm{CO}_{2}$ in six-well plate microplates. The medium was changed every 3 days during the incubation period. After 10 days, discarded the medium and washed 3 times. Then fixed the cells with $4 \%$ formaldehyde for $30 \mathrm{~min}$, and used $0.5 \%$ crystal violet stainning and observation.

\section{Transwell assay}

After transfection $48 \mathrm{~h}$, The SW620 cells were collected and resuspended. Carefully loaded it into a $8 \mu \mathrm{m}$ transwell chambers (Corning, USA), then placed the chamber into a 24-well plate. The cells were continue cultured $24 \mathrm{~h}$ and $48 \mathrm{~h}$ in serum-free medium. Used $4 \%$ paraformaldehyde fixing the cells $15 \mathrm{~min}$, and then stained it with crystal violet $25 \mathrm{~min}$ at $25^{\circ} \mathrm{C}$. Removed the suspension cells, imaged and counted the numbers of migrated onto the lower surface cells with an inverted microscope (Olympus, IX73) and photographed.2.9

\section{Flow cytometry}

The transfected SW620 cells were seeded into six-well plate at $1 \times 10^{6} /$ well per well. When the confluence rate reached more than $80 \%$, cell precipitate was collected. Fixed the cells with pre-prepared $70 \%$ frozen ethanol. Followed the protocol, stained the cells with AnnexinV and propidium iodide (PI) for $10 \mathrm{~min}$. Detected and analyzed the apoptosis cells with flow cytometer (BD Accuri C6).

\section{Western blots}

Harvested and lysed the cells with RIPA lysis buffer after transfection of $48 \mathrm{~h}$. Extracted

the total protein and fully denatured it in boiled water for $5 \mathrm{~min}$. Used BCA protein reagent kit(Thermofisher Scientific, Inc) detected the protein concentration. 10\% SDS-PAGE was used for the separation of the protein samples. After electrophoresis, the protein on SDS-PAGE was transferred onto PVDF membrane. $5 \%$ skim milk powder dilution was prepared with $10 \%$ TBST. Sealed the PVDE membrane with the defatted milk for $1 \mathrm{~h}$. Then incubated the membrane with specific primary antibodies at $4^{\circ} \mathrm{C}$ overnight. After that, incubated the PVDE membrane $1.5 \mathrm{~h}$ in secondary antibodies. Detected and analyzed The protein bands by Biolmaging Systems (BIO-RAD, USA). Use Anti-GAPDH antibody as an internal control.

\section{Drug experiments}

$100 \mathrm{uL}$ of untransfected SW620 cell suspension was added into 96-well plate (1X10 / well). When the cells adherent reached to $50 \% \bigotimes 60 \%$, added different concentration of PTX. The absorbance of each well 
at $24 \mathrm{~h}, 48 \mathrm{~h}$ and $72 \mathrm{~h}$ was observed by luminous detector (Glomax, Promega, 450nm wavelength). Based on the absorbance, the optimal PTX concentration and time (survival rate of 50\%) were calculated.

\section{Statistical analysis}

All the data statistics were used SPSS19.0 software. The results were expressed as mean value \pm SD of 3 or more determinations. T-test were applied to compare the differences between the two groups of data. Multiple groups data analysis were used one-way analysis of variance (ANOVA). It was statistically significant when $P<0.05$. All the experiments were performed at least with 3 individual repeat.

\section{Results}

\section{LncRNA CRNDE expression in colorectal cancer tissues and colorectal cancer cells.}

The differentially expressed genes were screened by microarray analysis. As shown in the volcano diagram 1A and cluster diagram 1B, LncRNA CRNDE was high expression in cancer tissues compared with paracancerous tissues. Different expression circRNAs between the CRC tissue and adjacent are displayed in the cluster analysis (Figure 1A). The volcano plot described the variation of circRNA expression between CRC and adjacent normal tissues (Figure 1B).

Further detected in 40 paired tisssues proved that LncRNA CRNDE was significantly up-regulated in colorectal cancer tissues, while its expression was relatively low in paracancerous tissues(Figure 1C). In addition, compared to the normal colorectal cell line NCM460, LncRNA CRNDE expression was significantly increased in four colorectal cancer cells HT116,SW620,SW480,LOVO(Figure 1D). Therefore, SW620 cells were selected for subsequent experiments.

To further clarify the function of LncRNA CRNDE in colorectal cancer cells, LncRNA CRNDE lentiviral vector being constructed. The vector was transfected into SW620 cell as empty vector group (NC), overexpressed group (OE-CRNDE) and interference group (Si-CRNDE). Fluorescent image and qRT-PCR results verified the expression of IncRNA CRNDE in different groups(Fig. 1E,F). The difference between the interference group and the over-expression group is significant $\left(*: P<0.05,{ }^{\star *} P<0.01\right)$.

\section{Over-expression of LncRNA CRNDE promoted the proliferation, invasion and migration of CRCs.}

CCK-8 assay proved that over-expression of LncRNA CRNDE increased the cell viability in the OE-CRNDE group, while the cell viability was significantly inhibited in the si-CRNDE group(Fig. 2A). Cloning formation experiments showed that over-expression of LncRNA CRNDE significantly increased the proliferation of SW620 cells (Fig. 2B). The wound healing assay confirmed that the migration ability of the OE-CRNDE group cells was significantly promoted, while the si-CRNDE group was significantly inhibited (Fig. 2C). 
Transwell assays results identified that the invasion of the CRC cells was enhanced in over-expression LncRNA CRNDE group. These data suggested that over-expression of LncRNA CRNDE can promote the proliferation, invasion and migration of CRCs.

\section{LncRNA CRNDE influenced CRCs cell cycle}

Based on the cell cycle results by flow cytometry anlysis, the S phrase and G2/M phrase cells was accumulating while G0/G1 phrase cells was decreasing in OE-CRNDE group(Fig. 3A). Western blot showed that the cell cycle related protein CDK4 and cyclinD1 expression was increased in OE-CRNDE group, while its expression in si-CRNDE group was decreased(Fig. 3B). The S phrase and G2/M phrase representated DNA replication and protein synthesis periods in cells, and was also the main period of cell division. The result proved that OE-CRNDE induced CRC cells division.

\section{LncRNA CRNDE promoted CRCs proliferation and migration through regulating the Wnt/ $\beta$-catenin signaling pathway}

Wnt/ $\beta$-catenin signaling pathway was a important pathway in the genesis and development of many cancer cells. Western blotting results showed that Wnt/ $\beta$-catenin related protein $\beta$-catenin, c-myc, APC, Axin2 in the OE-CRNDE group were increased, while the above proteins in the si-CRNDE group were significantly decreased (Fig. 4A). These results suggest that IncRNA CRNDE may affect SW620 proliferation and migration through the Wnt/ $\beta$-catenin signaling pathway.

\section{LncRNA CRNDE induced CRC cells resisitance to PTX}

Previous research have reported the treatment of PTX in cancer, but the mechanisim invovled in the process still not clear. To further revealed the mechanisim of PTX treatment to CRC, we first treated SW620 cells with different concentrations of PTX to identified the optimal drug concentration and time. Based on the results, we selected $0.06 \mathrm{~g} / \mathrm{uL}$ as the PTX treat concentration dor next experiment.

After that, transfected OE-CRNDE and si-CRNDE vector into SW620 cells, then treated the cells with PTX. Wound healing assay showed that the proliferation of the cells was accelerated in OE-CRNDE group after treated with PTX(Fig. 5A). Transwell experiment proved that when treated with PTX, the cell invasion and migration ability in the OE-CRNDE group was increased than that in the NC group, while it was decreased in the si-CRNDE group(Fig. 5B). Cell cycle experiment showed that when treated with PTX in OE-CRNDE group, the G0/G1 phase cells was shortan while G2/M phase cells was longer than that in other groups. These results suggested that IncRNA CRNDE could enhance the resisitance of CRC to PTX, thus affecting the proliferation and invasion of CRC (Fig. 5C).

\section{LncRNA CRNDE enhanced CRC resisitance to PTX through Wnt/ $\beta$-catenin signaling pathway}

To further confirm the mechanisims of IncRNA CRNDE regulation CRC to paclitaxel treatment, appropriate amount of PTX was added to different CRCs groups. After a period of culture, the protein expression was 
analyzed by western blot. Compared to the control group, the cell cycle related protein CDK4 and cyclinD1 was significantly decreased both in OE-CRNDE and si-CRNDE group after treated with PTX(Fig. 6A). The

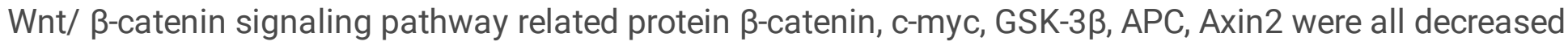
when treated the with PTX(Fig. 6B).These results suggested that over-expression of IncCRNDE could enhance the $\mathrm{CRC}$ resisitance to PTX through inhibited the Wnt/ $\beta$-catenin signaling pathway.

\section{Discussion}

Lnc RNA was a group a non coding RNA which involved in several bio-functions of different tumor. IncRNA CRNDE as a regulate gene often plays important function in cancer cells proliferation and matastasis[16]. Previous research have reported that IncRNA CRNDE was dysregulated in bladder cancer[17], renal cell carcinoma[18], gastric cancer, breast cancer and osteosarcoma[19]. By analyzed CRC tissue specimens with microarray and RT-PCR assays, LD. Graham et al reported IncRNA CRNDE was one of the significant tissue and plasma biomarkers for colorectal adenomas and cancers[20]. Wang et al reported that the glioma cells growth and invasion was promoted by over-expression of IncRNA CRNDE, and the IncRNA CRNDE positively correlates with EGFR activation[21]. Luo et al found that IncRNA CRNDE promoted glioblastoma progression through sponge to mir-9-5p[22]. Shi et al reported that IncRNA-CRNDE promoted BMSC chondrogenic differentiation and cartilage repair through SIRT1/SOX9[23]. In this study, we found that IncRNA CRNDE was high expressed in CRC tissues. The high expression of IncRNA CRNDE promoted migration and invasion ability of CRC, while interference of CRNDE significantly inhibited the cells proliferation and colony formation.

Wnt/ $\beta$-catenin signaling pathway is an important pathway in the genesis and development of many cancer cells. The pathway consisited with wnt, $\beta$-catenin, Axin2, APC and glycogen synthase kinase $3 \beta$ (GSK-3 $\beta$ ) [24]. Wnt/ $\beta$-catenin signaling pathway can be divided into typical( $\beta$-catenin dependent) and atypical types( $\beta$-catenin independent). When the signaling pathway is inactive, $\beta$-catenin is phosphorylated and degraded by a destruction complex, which contains the scaffold protein Axin2, APC and the kinases GSK3 $\beta[25]$. When the pathway is activated, the destruction complex is inactivate, leads to a translocation of $\beta$-catenin into nucleus and to activate the downstream targets. Moreover, activated $W n t / \beta$-catenin signaling pathway also associated with cancer stem cells proliferation and therapeutic resistance[26]. In this study, we found that the $\beta$-catenin, c-myc, APC and Axin2 protein were increased in the OE-CRNDE group, while the above proteins were decreased in the si-CRNDE group.

PTX is a tricyclic diterpenoid compound which often used as a natural anticancer drugs in cancer treatments[27]. PTX can promote the tubulin assembly into microtubules and prevent the microtubules dissociation.Therefor, PTX inhibited cancer cells growth by blocking the cell cycle progression and preventing its mitosis[28]. The treatment of PTX was regulated by several factors, IncRNA as a regulate gene also plays important role in the process. Yang et al reported that propofol could enhanced the prostate cancer cells sensitivity to PTX[29]. Gu et al showed that LncRNA NONHSAT141924 could promote paclitaxel chemotherapy resistance through $\mathrm{p}-\mathrm{CREB} / \mathrm{Bcl}-2$ apoptosis signaling pathway in breast cancer[30]. The results suggests that LncRNA NONHSAT141924 might be an efficient therapeutic 
strategy for PTX-resistant breast cancer treatment. But there are no any research about IncRNA CRNDE and PTX resistance.

In this study, we first treated and identified SW620 cells sensitivity to PTX. Then, transfected OE-CRNDE and si-CRNDE into SW620 cells and treated the cells with PTX. Transwell experiment proved that the ability of cell invasion and migration in the OE-CRNDE group was increased than that in the NC group, while it was decreased in the si-CRNDE group. Cell cycle experiment proved that when treated with PTX, the $S$ phase cells of OE-CRNDE group was longer than that in the untreated group, and the extension was most significant. These results suggested that IncRNA CRNDE could enhance the sensitivity of CRC cells to PTX, thus affecting the proliferation and invasion of CRC.

To further reveal the mechanisim of IncRNA CRNDE regulation PTX treatment CRC, The cell cycle and $\mathrm{Wnt} / \beta$-catenin signaling pathway related protein were analyzed with western bloting. Compared to the control group, the cell cycle related protein CDK4 and cyclinD1 was significantly decreased both in OECRNDE and si-CRNDE group after treated with PTX. The Wnt/ $\beta$-catenin signaling pathway related protein $\beta$-catenin, c-myc, GSK-3 $\beta$, APC and Axin2 were all increased in OE-CRNDE group,while their all decreased when treated with PTX. These results suggested that over-expression of IncCRNDE could enhance the $\mathrm{CRC}$ resisitance to PTX through inhibited the Wnt/ $\beta$-catenin signaling pathway.

\section{Conclusions}

In brief, our study revealed that IncRNA CRNDE promotes the proliferation, invasion and migration of CRCs. Over-expression of LnCRNA CRNDE enhanced the resisitance of CRC to PTX through inhibited of Wnt/ $\beta$-catenin signaling pathway. The results also demostrated that targeting IncRNA CRNDE may present a reliable therapeutic target for CRC. Further research about IncRNA CRNDE in CRC is needed for validation.

\section{Declarations}

\section{Authors' contributions}

Li-bin Wang provided the experimental design; Rui Ma and Li-bin Wang wrote the draft of the manuscript; Chuan-yang Yu. Xiang Tao and Zhi Yang, Qi Huang, Pei-dong You performed the experiments; Jin-hai Tian, Jia Cao and Jie Cao analyzed the data; Li-bin Wang prepared all figures, Rui Ma, Li-bin Wang, Chuan-yang Yu wrote, reviewed and edited the manuscript.The author(s) read and approved the final manuscript.The authors declares that there are no conflict of interest exists in the paper.

\section{Funding}

This study was supported by the National Natural Science Foundation of China (No. 81860470); Special talent startup program of Ningxia Medical University (Grant No. XT2017017); The Ningxia Key R\&D Programs (Grant No.2019BFH02012); The Ningxia Biochip Technology Research and Development 
Innovation Team (Grant No.2019-18); Ningxia high level science and technology innovation leading talent project (Grant No. KJT2019003); The Scientific Research Platform Open Project of the General Hospital of Ningxia Medical University (Grant No.2020-146).

\section{Availability of data and materials}

All data and plasmid constructs generated during the current study are available from the corresponding author on reasonable request.

\section{Ethics approval and consent to partecipate}

The research were approved by the Ningxia Medical University General Hospital Scientific Research Ethics Committee (Ethics No. 2019-175). All test methods were performed in accordance with the guidelines of ethical requirements. The informed consents were signed from all participants involved in the study.

\section{Consent for publication}

Not Applicable

\section{References}

1. Sung H, Ferlay J, Siegel RL, Laversanne M, Soerjomataram I, Jemal A, Bray F. Global Cancer Statistics 2020: GLOBOCAN Estimates of Incidence and Mortality Worldwide for 36 Cancers in 185 Countries. CA Cancer J Clin. 2021;71(3):209-249.

2. Lightdale CJ. Colorectal Cancer Screening: Where We Are and Moving Forward. Gastrointest Endosc Clin N Am. 2020; 30(3):xiii-xiv.

3. Gini A, Jansen EEL, Zielonke N, Meester RGS, Senore C, Anttila A, Segnan N, Mlakar DN, de Koning HJ, Lansdorp-Vogelaar I; EU-TOPIA consortium. Impact of colorectal cancer screening on cancerspecific mortality in Europe: A systematic review. Eur J Cancer. 2020;127:224-235.

4. Xie S, Chang Y, Jin H, Yang F, Xu Y, Yan X, Lin A, Shu Q, Zhou T. Non-coding RNAs in gastric cancer. Cancer Lett. 2020;493:55-70.

5. Chi Y, Wang D, Wang J, Yu W, Yang J. Long Non-Coding RNA in the Pathogenesis of Cancers. Cells. 2019;8(9):1015.

6. Sanchez Calle A, Kawamura Y, Yamamoto Y, Takeshita F, Ochiya T. Emerging roles of long noncoding RNA in cancer. Cancer Sci. 2018;109(7):2093-2100.

7. Lu Y, Sha H, Sun X, Zhang Y, Wu Y, Zhang J, Zhang H, Wu J, Feng J. CRNDE: an oncogenic long noncoding RNA in cancers. Cancer Cell Int. 2020;20:162-172.

8. Tang D, Zhao L, Peng C, Ran K, Mu R, Ao Y. LncRNA CRNDE promotes hepatocellular carcinoma progression by upregulating SIX1 through modulating miR-337-3p. J Cell Biochem. 2019 Sep;120(9):16128-16142. 
9. Bai X, Wang W, Zhao P, Wen J, Guo X, Shen T, Shen J, Yang X. LncRNA CRNDE acts as an oncogene in cervical cancer through sponging miR-183 to regulate CCNB1 expression. Carcinogenesis. 2020 Mar 13;41(1):111-121.

10. Han P, Li JW, Zhang BM, Lv JC, Li YM, Gu XY, Yu ZW, Jia YH, Bai XF, Li L, Liu YL, Cui BB. The IncRNA CRNDE promotes colorectal cancer cell proliferation and chemoresistance via miR-181a-5p-mediated regulation of Wnt/ $\beta$-catenin signaling. Mol Cancer. 2017;16(1):1-14.

11. Cheng $X, X u X$, Chen D, Zhao F, Wang W. Therapeutic potential of targeting the Wnt/ $\beta$-catenin signaling pathway in colorectal cancer. Biomed Pharmacother. 2019 Feb;110:473-481.

12. Huang JQ, Wei FK, Xu XL, Ye SX, Song JW, Ding PK, Zhu J, Li HF, Luo XP, Gong H, Su L, Yang L, Gong LY. SOX9 drives the epithelial-mesenchymal transition in non-small-cell lung cancer through the Wnt/ ß-catenin pathway. J Transl Med. 2019 May 6;17(1):143-157.

13. Chen M, Li L, Zheng PS. SALL4 promotes the tumorigenicity of cervical cancer cells through activation of the Wnt/ $\beta$-catenin pathway via CTNNB1. Cancer Sci. 2019 Sep;110(9):2794-2805.

14. Zhu L, Chen L. Progress in research on paclitaxel and tumor immunotherapy. Cell Mol Biol Lett. 2019 Jun 13;24:40-51.

15. Yang YH, Mao JW, Tan XL. Research progress on the source, production, and anti-cancer mechanisms of paclitaxel. Chin J Nat Med. 2020 Dec;18(12):890-897.

16. Cheng J, Chen J, Zhang X, et al. Overexpression of CRNDE promotes the progression of bladder cancer. Biomed Pharmacother. 2018;99:638-644.

17. Cheng J, Chen J, Zhang X, et al. Overexpression of CRNDE promotes the progression of bladder cancer. Biomed Pharmacother. 2018;99:638-644.

18. Zhang Y, Lan X, Wang Y, et al. CRNDE mediates the viability and epithelial-mesenchymal transition of renal cell carcinoma via miR-136-5p. Journal of Receptor and Signal Transduction Research, 2020(1):1-11.

19. Yu Y, Wang L, Li Z, et al. Long noncoding RNA CRNDE functions as a diagnostic and prognostic biomarker in osteosarcoma, as well as promotes its progression via inhibition of miR-335-3p. Journal of Biochemical and Molecular Toxicology, 2021; 35(5):e22734.

20. Graham LD, Pedersen SK, Brown GS, et al. Colorectal neoplasia differentially expressed (CRNDE), a novel gene with elevated expression in colorectal adenomas and adenocarcinomas. Genes Cancer. 2011;2(8):829-840.

21. Wang Y, Wang Y, Li J, Zhang Y, Yin H, Han B. CRNDE, a long-noncoding RNA, promotes glioma cell growth and invasion through mTOR signaling. Cancer Lett. 2015;367:122-128.

22. Luo X, Tu T, Zhong Y, Xu S, Chen X, Chen L, Yang F. ceRNA Network Analysis shows that IncRNA CRNDE Promotes Progression of Glioblastoma through sponge mir-9-5p. Front Genet. 2021;12:617350.

23. Shi C, Zheng W, Wang J. IncRNA-CRNDE regulates BMSC chondrogenic differentiation and promotes cartilage repair in osteoarthritis through SIRT1/SOX9[J]. Molecular and Cellular Biochemistry, 2021;476(4):1881-1890.

Page $11 / 19$ 
24. Chatterjee $A$ „, Paul $S$, Bisht $B$, et al. Advances in targeting the WNT/ $\beta$-catenin signaling pathway in cancer[J]. Drug Discovery Today, 2021; S1359-6446 (21):317-321

25. Anthony CC, Robbins DJ, Ahmed Y, Lee E. Nuclear Regulation of Wnt/ $\beta$-Catenin Signaling: It's a Complex Situation. Genes (Basel). 2020;11(8):886-898.

26. Hu XY, Hou PF, Li TT, Quan HY, Li ML, Lin T, Liu JJ, Bai J, Zheng JN. The roles of Wnt/ $\beta$-catenin signaling pathway related IncRNAs in cancer. Int J Biol Sci. 2018;14(14):2003-2011.

27. Alves RC, Fernandes RP, Eloy JO, Salgado HRN, Chorilli M. Characteristics, Properties and Analytical Methods of Paclitaxel: A Review. Crit Rev Anal Chem. 2018;48(2):110-118.

28. Ghafouri-Fard S, Shoorei H, Abak A, Abbas Raza SH, Pichler M, Taheri M. Role of non-coding RNAs in modulating the response of cancer cells to paclitaxel treatment. Biomed Pharmacother. 2021;134:111172.

29. Yang X, Qin J, Gong C, et al. Propofol enhanced the cell sensitivity to paclitaxel (PTX) in prostatic cancer (PC) through modulation of HOTAIR[J]. Genes \& Genomics, 2021, 43(7):807-814.

30. Zhao F, Yang Z, Gu X, et al. miR-92b-3p Regulates Cell Cycle and Apoptosis by Targeting CDKN1C, Thereby Affecting the Sensitivity of Colorectal Cancer Cells to Chemotherapeutic Drugs[J]. Cancers, 2021,13(13):3323.

\section{Figures}


A

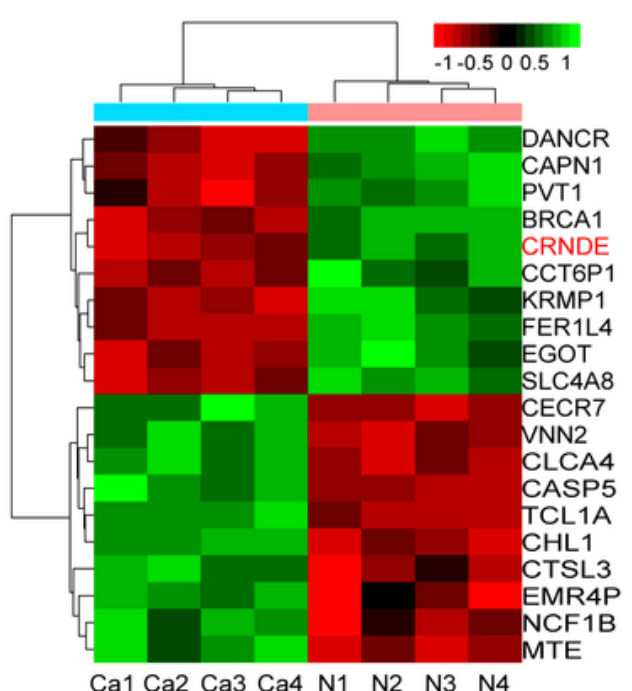

C

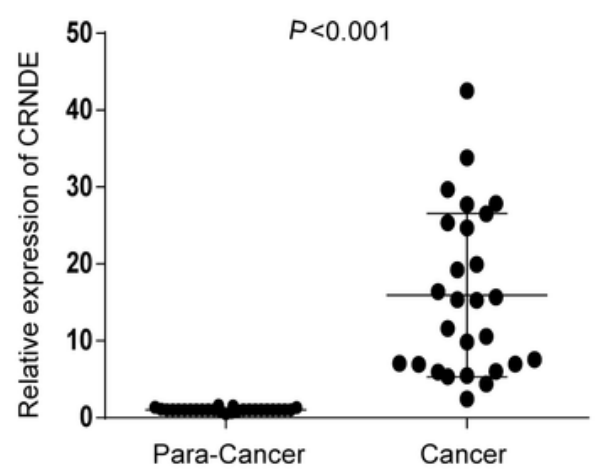

E

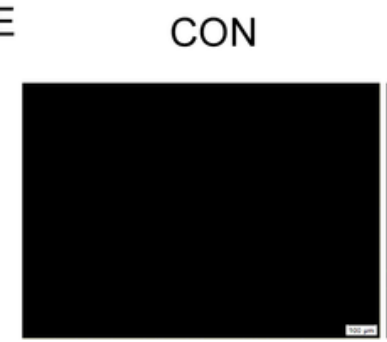

OE-CRNDE

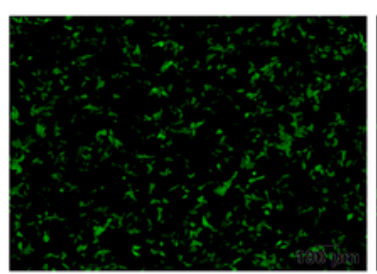

B

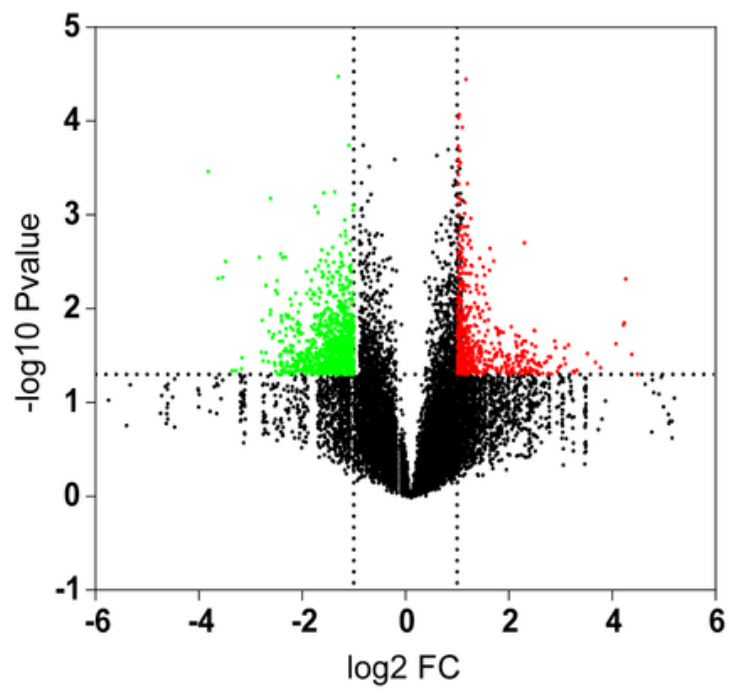

D

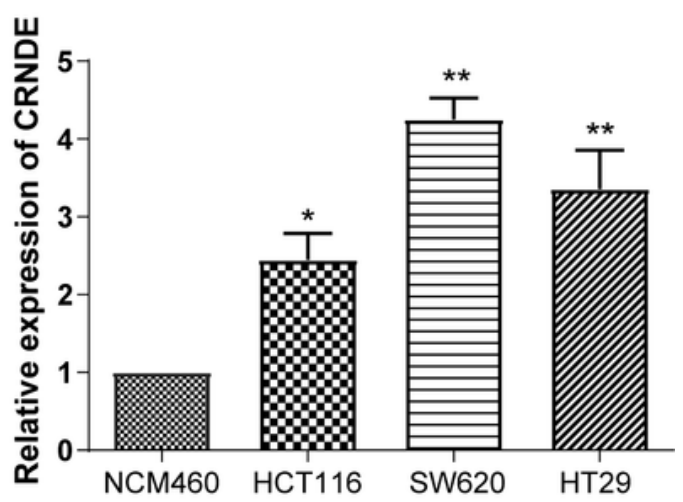

F

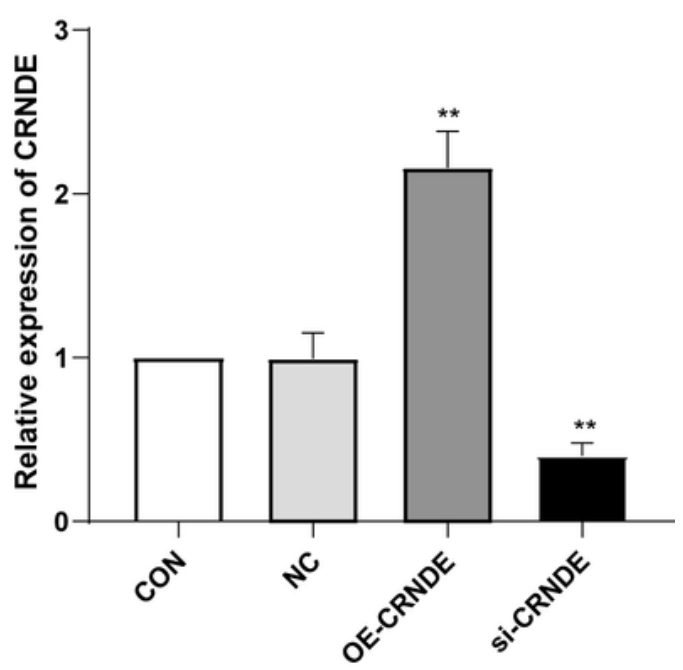

\section{Figure 1}

Identification of IncRNA CRNDE expression in colorectal cancer tissues and cells. A,B: Volcano diagram and cluster diagram demonstrated the IncRNA Microarray analysis results between colorectal cancer and normal tissues. C: Relative expression of IncRNA CRNDE in colorectal cancer and para-cancer tissues (怄

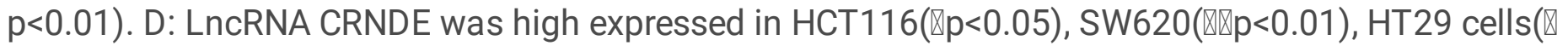


$p<0.05)$. E,F: Fluorescent image and qRT-PCR results verified the expression of IncRNA CRNDE in different groups $(\mathbb{\otimes} \mathrm{p}<0.01)$.

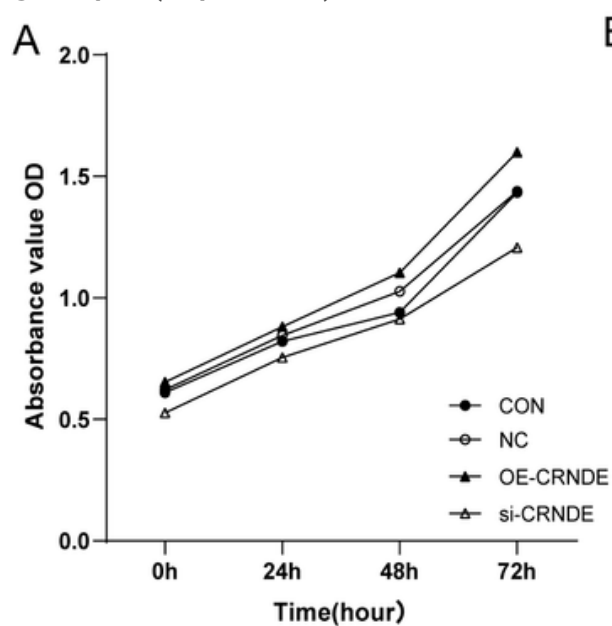

C

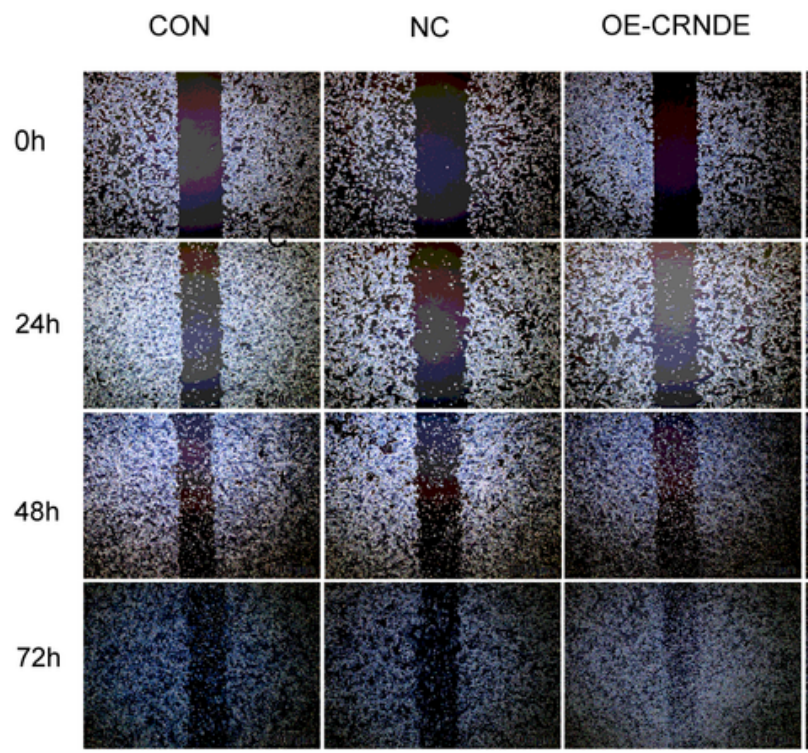

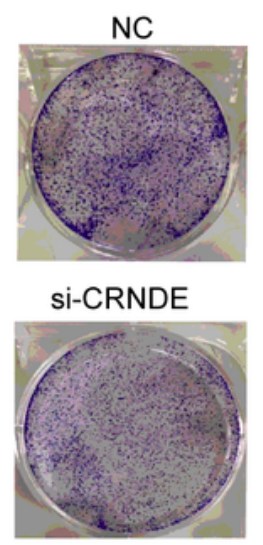

si-CRNDE
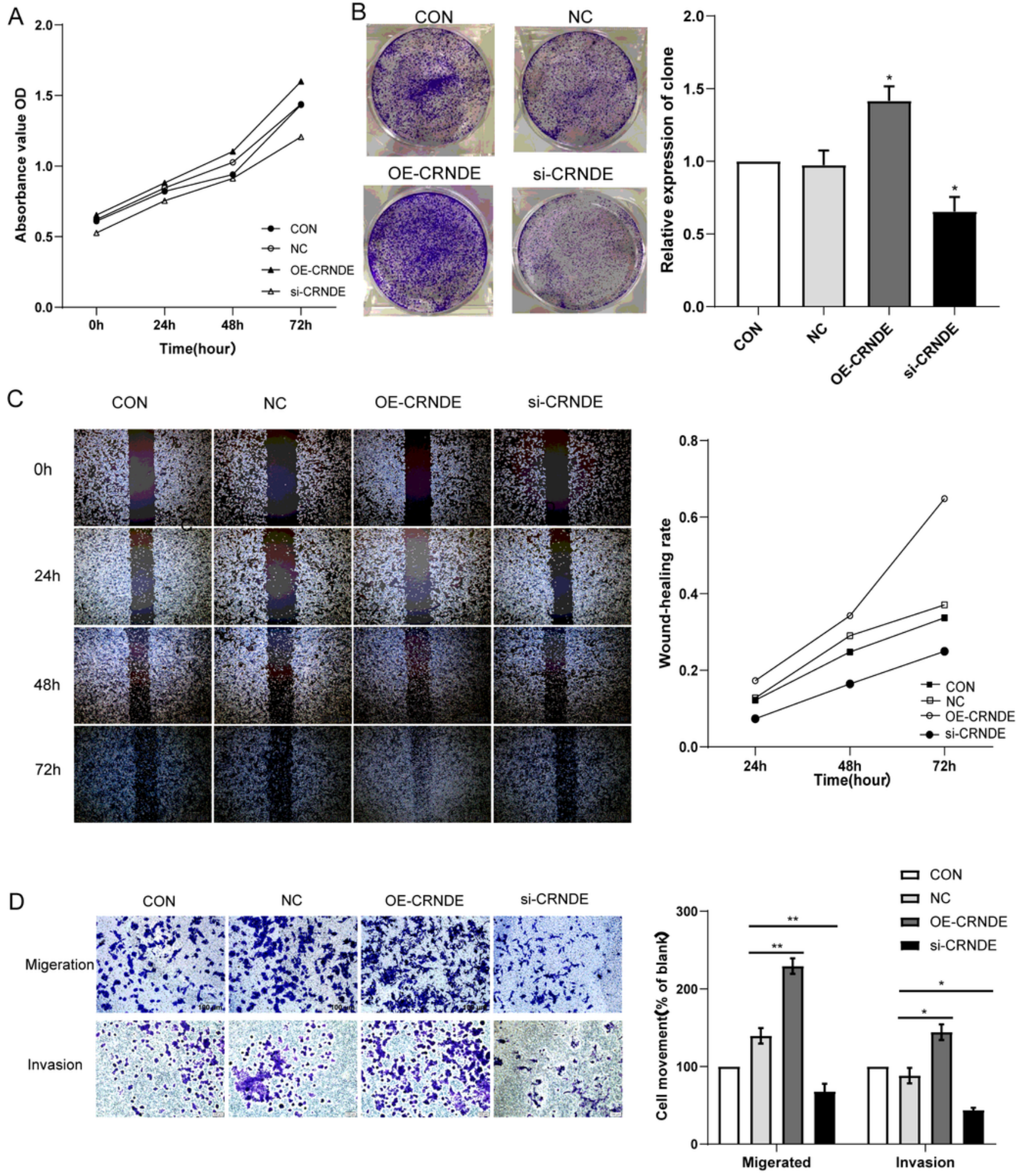

Figure 2

Over-expression of LncRNA CRNDE promoted colorectal cancer cells proliferation,migration and invasion. A,B: CCK8 assay and cloning formation experiments showed that over-expression of LncRNA CRNDE significantly increased the proliferation of $S W 620$ cells $(\llbracket p<0.05)$. C: Wound healing assays from Oh to 
$72 \mathrm{~h}$ following the transfection proved that over-expression of LncRNA CRNDE enhanced SW620 cells migration. D: Transwell assays results identified that the invasion of over-expression of LncRNA CRNDE cells was increased( $(\mathrm{p} p<0.05, \mathbb{\text { , }} \mathrm{p} p<0.01)$.

A
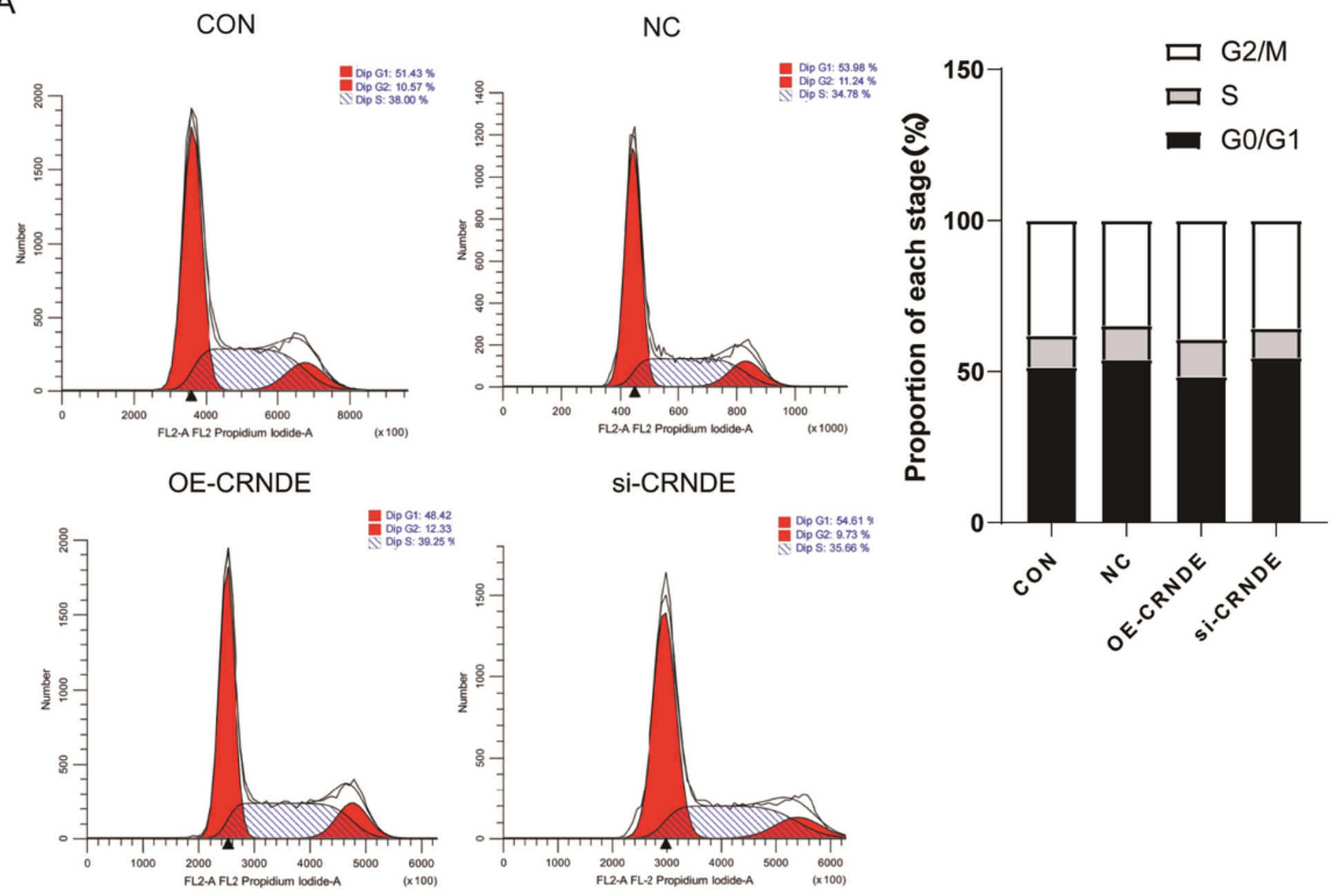

B
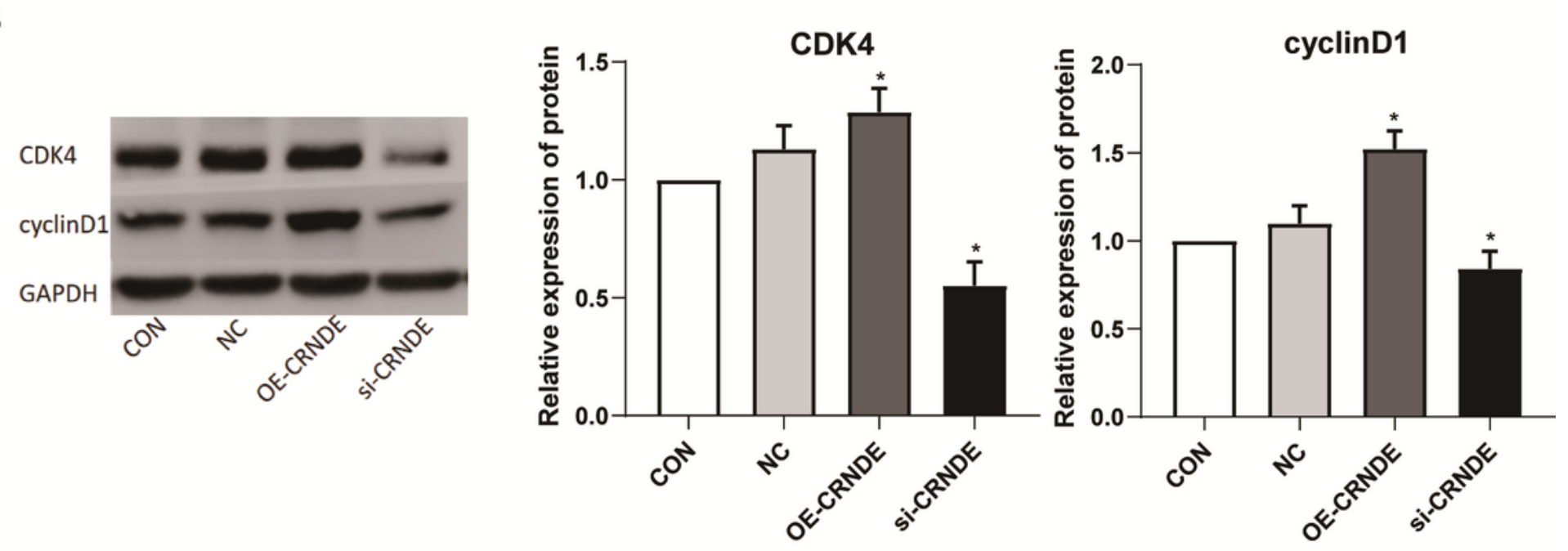

Figure 3

LncRNA CRNDE influenced CRCs cell cycle. A: The G0/G1 phase SW620 cells was lower in OE-CRNDE group while $S$ phase and G2/M phase cells was higher than that of nc and si-CRNDE group. B: Western 
blot proved the relative expression of CDK4 and CyclinD1 protein in OE-CRNDE group was significantly

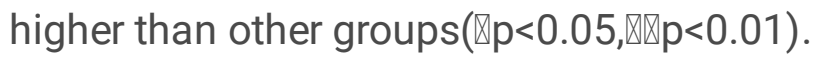

A
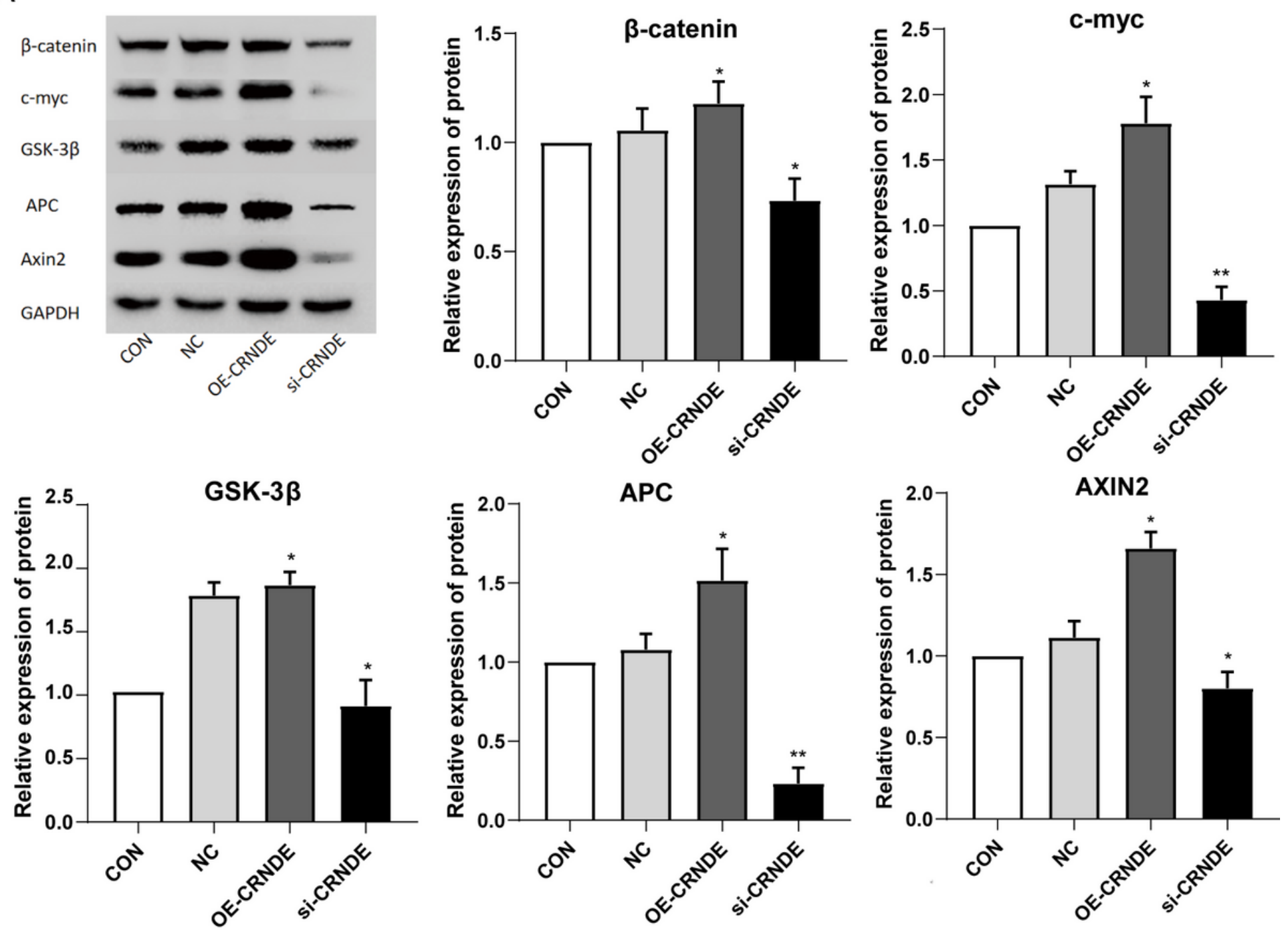

Figure 4

LncRNA CRNDE promoted Wnt/ $\beta$-catenin signaling pathway related proteins expression in CRCs A: following the transfection of OE-CRNDE and si-CRNDE vector, the expresion of Wnt/ $\beta$-catenin signaling pathway related protein $\beta$-catenin, c-myc, APC, Axin2 were increased compared with those in the NC group

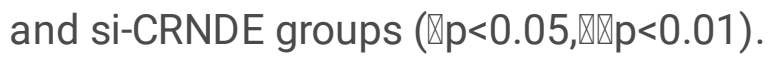



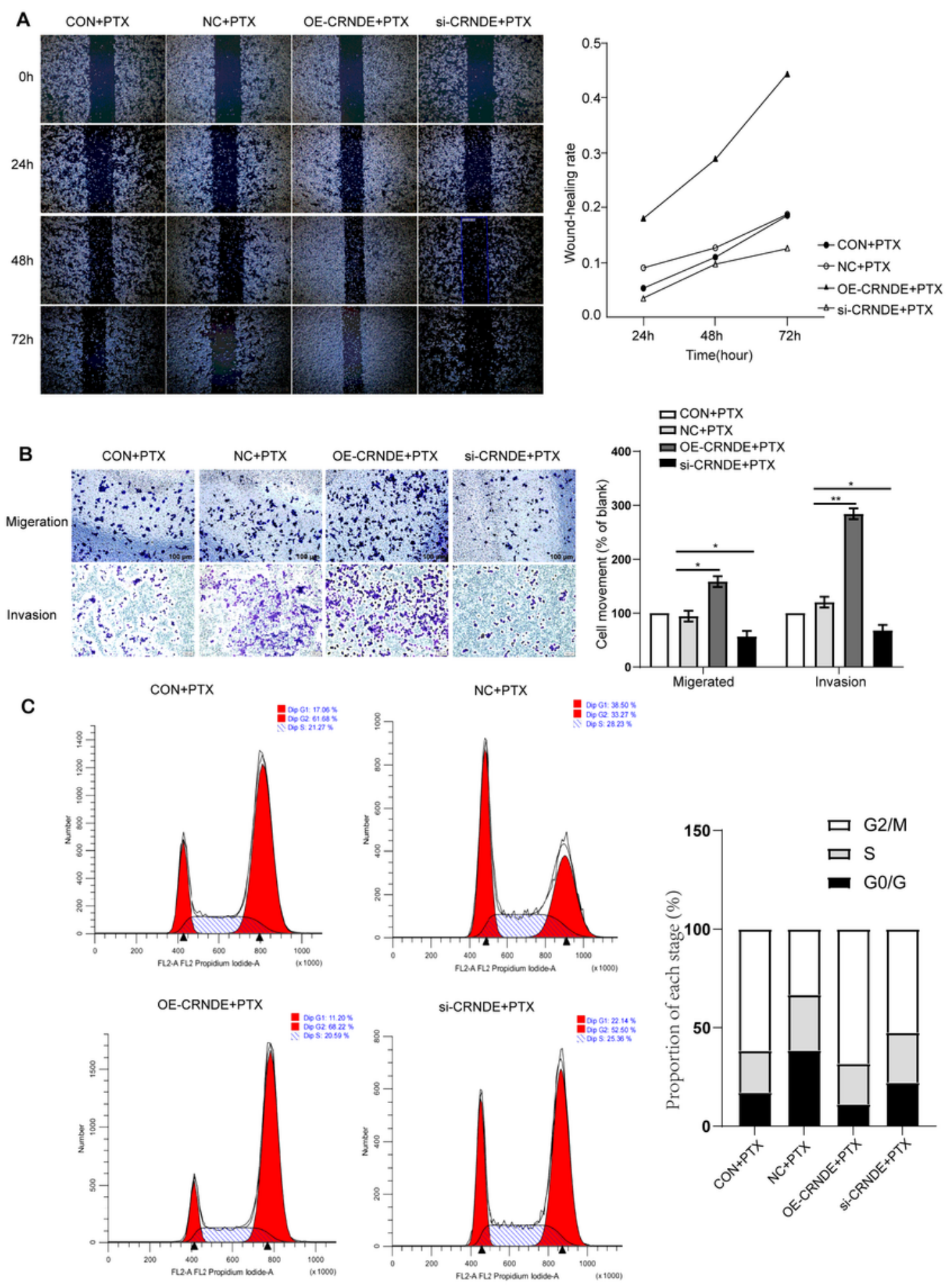

\section{Figure 5}

LncRNA CRNDE enhanced CRC resisitance to PTX through Wnt/ $\beta$-catenin signaling pathway. A: Wound healing assay showed that the proliferation of the cells was accelerated in OE-CRNDE group after treated with PTX. B:Transwell experiment proved that when treated with PTX, the cell invasion and migration ability in the OE-CRNDE group was increased than that in the NC group, while it was decreased in the si- 
CRNDE group. C: when treated with PTX in OE-CRNDE group, the G0/G1 phase cells was shortan while $\mathrm{G} 2 / \mathrm{M}$ phase cells was longer than that in other groups. $(\llbracket p<0.05, \mathbb{\otimes} \mathrm{p}<0.01)$.

\section{A}

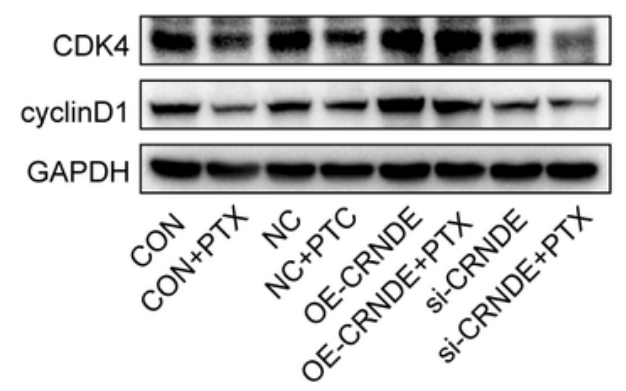

B
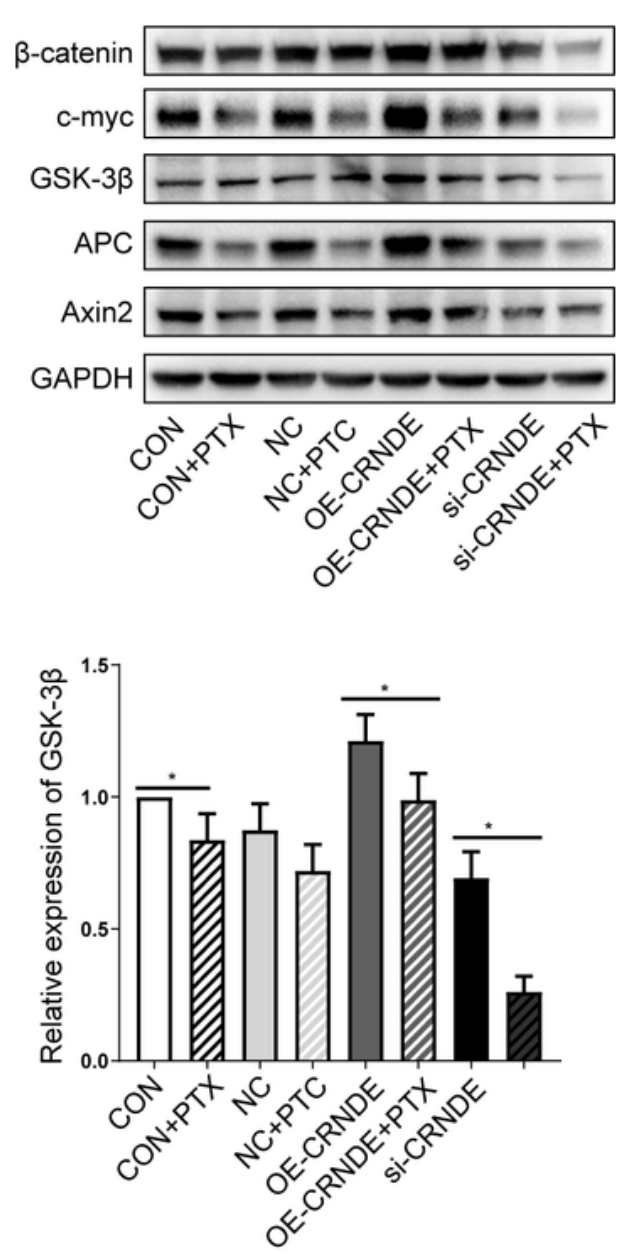
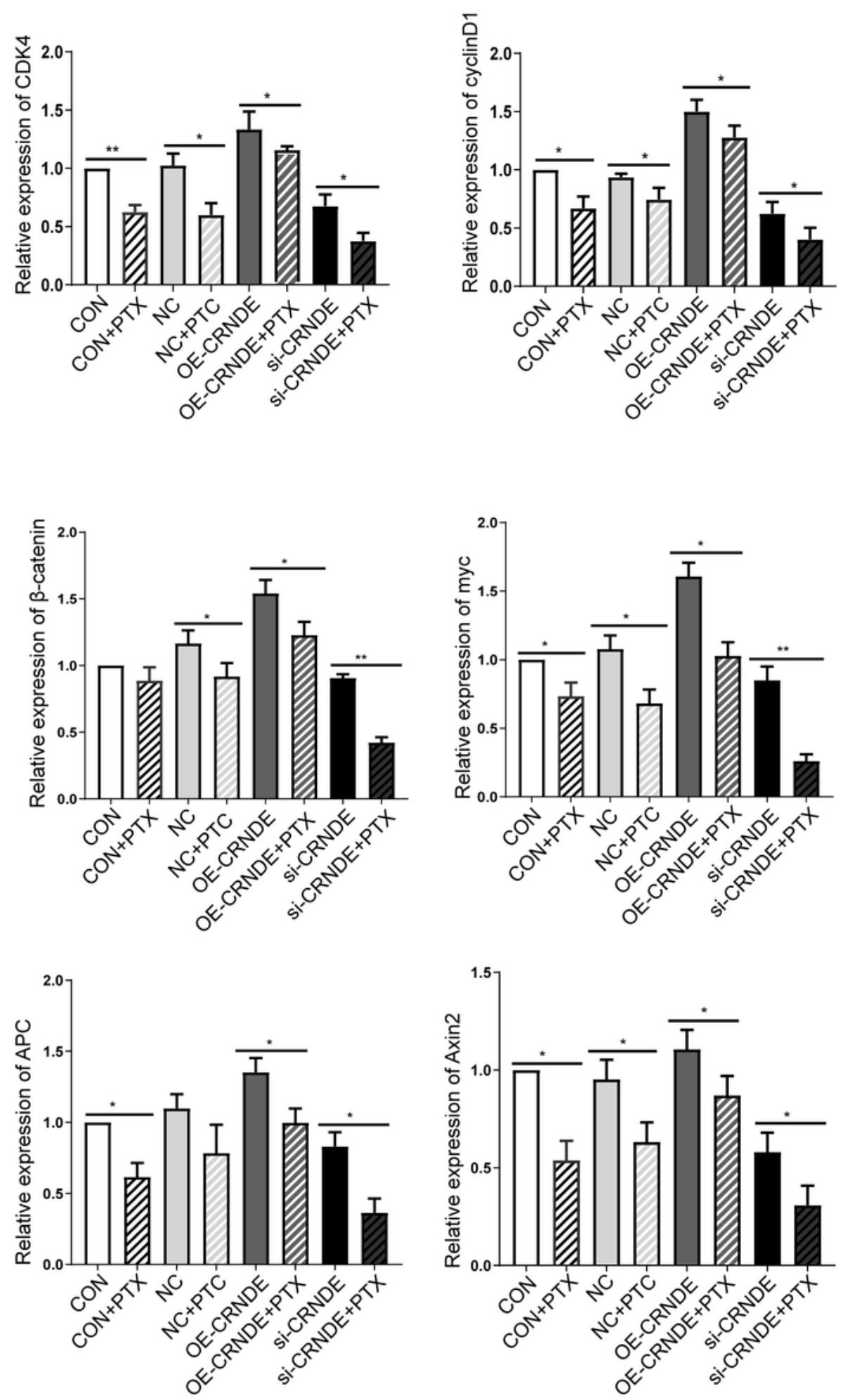

Figure 6

LncRNA CRNDE enhanced CRC resisitance to PTX was regulated by Wnt/ $\beta$-catenin signaling pathway A: After PTX treated the expression of CDK4 and CyclinD1 protein were all decreased in OE-CRNDE and si- 
CRNDE group. B: The expression of $\beta$-catenin, c-myc, GSK-3 $\beta$, APC, Axin2 proteins were all decreased when treated with PTX in different groups. $(\llbracket p<0.05, \mathbb{Q} \mathbb{p}<0.01)$. 